\title{
Diagnosis of prostate cancer in one day: The benefits of cytology in tumour detection
}

$\underline{\text { Eva Compérat }} 1 \stackrel{2}{2}, \underline{\text { Raphaele Renard-Penna }} 1 \stackrel{1}{3}$, Valérie Ondet 1 , Géraldine Cancel-Tassin 1 , Olivier Cussenot $1 \frac{1}{4}$

${ }^{1}$ GRC5 predictive onco-urology, Sorbonne University, Paris, France.

2 Department of Pathology, Hopital Tenon, Sorbonne University, Paris, France.

${ }^{3}$ Department of Radiology, Hopital La Pitié Salpêtrière, Sorbonne University, Paris, France.

${ }^{4}$ Department of Urology, Hopital Tenon, Sorbonne University, Paris, France.

\begin{abstract}
Introduction

Prostate cancer (PCa) is a frequent and mortal disease. The aim of the study was to accelerate with the help of prostate cytology the handling of PCa patients by giving results within 3 hours. Standard histology served as control. The aim was to introduce a « diagnosis and handling of $\mathrm{PCa}$ in one day » concept.
\end{abstract}

\section{Material and methods}

After multiparametric MRI, prostate biopsies were taken and one of the biopsies was used for cytology on superfrost slides. The cytology samples were stained by p63/p504s double staining, a standard stain in PCa histology, interpretation took place within 3 hours.

\section{Results}

Among 129 patients $39.5 \%$ had a prior history of $\mathrm{PCa}$ and were either under active surveillance or had been treated by focal therapy. The others came with suspicion of PCa. In $80.8 \%$ the cytology and histology results were concordant. In low grade PCa the detection was more difficult with $72.4 \%$ of detection, in high grade $\mathrm{PCa}$ the result were according to the differentiation between 81.8 and $90 \%$. False positive cases were less than $4.0 \%$.

\section{Conclusion}

Cytology of the prostate is unusual, our study is the first to show that prostate cytology is feasible and gives satisfying immediate results, especially in more aggressive disease. Immunocytology can be easily integrated into the laboratory. Our technique allows a quicker handling of PCa and allows to soften the psychological impact on men waiting for the diagnosis of $\mathrm{PCa}$. 
Keywords : Prostate cancer, cytology, immunocytology, p63, racemase

'Data availability statement' : The data that support the findings of this study are available from the corresponding author upon reasonable request. 


\section{Introduction}

Prostate cancer $(\mathrm{PCa})$ is a major concern in public health and more than 1.1 million cases worldwide are detected every year (1). Although treatments are variable and have considerably changed, metastatic disease still exists and the mortality is relatively high with 26730 estimated deaths in 2017 (2). It is important to take care of PCa patients quickly, as the prognosis depends on quick handling, but even if the tumor is not aggressive, the psychological impact for the individual patient is important, therefore starting a treatment as early as possible is necessary.

The idea of a "one - day diagnosis" is a recent concept coming from breast cancer, in which the clinicians set up a patients' circuit during one day. Within one day, the radiologist, cytologist/pathologist and all the following medical consultations (surgeon, oncologist, psychologist) are set up in order to allow a rapid patient's handling (3).

The idea of « PCa handling in one day » was to organise a circuit for patients with either suspicion of PCa or patients under active surveillance of PCa. Our approach in detecting and follow-up of PCa is entirely new, and our team is the first one to explore this one-day diagnosis of PCa. The first step was to perform a multiparametric MRI (mpMRI) followed by prostate biopsies. On the biopsy material, cytology was made with the help of appositions. The cytology sample was immediately stained by immunocytology and the interpretation took place around 3 hours later. The final histology report of prostate biopsies served as control. One of the advantages of handling PCa in one day is that all the specialists are on place and the working chain is extremely efficient. Concertation amongst all involved medical specialists is easy.

The advantage of immunocytology on prostate tissue is, that the basal cells as well as the tumor cells can easily be stained by immunohistochemistry: p63 stains the nucleus of basal cells that are present in normal prostate tissue but not anymore in PCa, whereas, the cytoplasm from the tumor cells can be stained by a p504s (racemase)antibody. Therefore, interpretation of immuncytology is relatively easy even on cytology samples.

For a better understanding, it must be underlined that recent consensus papers which were integrated into the latest WHO classification 2016 (4) decided that Gleason score should be given in grade groups: Gleason $6(3+3)$, which is the lowest grade and shows the best differentiation is considered ISUP grade group 1, Gleason $7(3+4)$ as ISUP grade group 2, Gleason $7(4+3)$ as ISUP grade group 3. Underlining the difference between the two Gleason scores $7(3+4)$ and $(4+3)$ is very important, as it is well known that the second displays more aggressive features and should not be followed by active surveillance. Furthermore, Gleason 
score $8(4+4),(3+5)$ or $(5+3)$ are considered ISUP grade group 4 and last but not least Gleason score 9 and $10(4+5),(5+4)$ or $(5+5)$ are in ISUP grade group 5. ISUP grade group 1 can be considered as low aggressive PCa, highly aggressive are scores between 8-10 ISUP grade group 4 and 5, and intermediate are ISUP grade group 2 and 3 . The aim of the study was double, first, to see whether this handling is feasible and if the results from cytology differ from the definitive result by standard histology. Second, whether this quick handling is helpful in the patient's circuit. 


\section{Material and Methods}

\section{Patients}

All included patients were instructed according to ethical standards and agreed on the protocol. The patients came to our centre, which is a reference centre for $\mathrm{PCa}$, either with suspicion of $\mathrm{PCa}$ (increased levels of PSA, urinary trouble, induration of the prostate on clinical examination), or with an already diagnosed $\mathrm{PCa}$ under active surveillance but with increasing PSA.

\section{MRI and Prostate Biopsy}

First patients underwent a multiparametric prostate MRI (mpMRI). The interpretation of the multiparametric MRI was done at the local site by a single senior uroradiologist, who had access to clinical details. The quality of the mpMRI was checked at the start of the study. The radiologist assessed clinically significant PCa using the PIRADS score, all patients with a PIRADS score from 3-5 underwent prostate biopsies. The transrectal ultrasound-guided prostate biopsy was done by the senior uroradiologist under local anaesthesia. In case patients had a target on MRI, they underwent only target biopsies, all the others had 12 standard biopsies and eventual additional target biopsies in case of suspicion of an eventual mpMRI target.

Then one of the standard biopsies or target biopsies on imaging was chosen to make several appositions on a superfrost slide. Only superfrost slides were used. The biopsy was put on the slide with the help of a forceps, several appositions were made, according to clinical cytological standards.

In the meantime, the prostate biopsy, which was used for cytology, was fixed in formalin (minimal fixation duration was 6-10 hours), and underwent the normal technical circuit like all biopsy material in our lab to be read the next day.

\section{Immunocytology}

The slide was then dried for 30 minutes at ambient temperature. The next step was to fix the sample with acetone for 10 minutes at $4^{\circ} \mathrm{C}$. After this step, the slide was dried another 30 minutes at room temperature. No pre-treatment was done on the slide, which was then put into the stainer (Bench Mark Ultra Roche, series 311230) for 1 hour and 12 minutes. A double staining with the following mouse monoclonal antibodies was undertaken: p63 (Dako M7317, dilution 1/200) and racemase (Dako M3606 dilution 1/200), clone $13 \mathrm{H} 4$. The counterstaining was done with hemateine for 8 minutes. 


\section{Interpretation criteria}

The slides were read by a senior pathologist specialised in uropathology. For the interpretation, the following criteria were taken into consideration: cytoplasmic staining of epithelial cells by p504s (racemase) and nuclear staining of basal cells by p63.

The possible answers on the cytology were as follows: 1) Negative for PCa: nuclear staining of basal cells and no cytoplasmic staining of epithelial cells, 2) suspicious for PCa: staining of both nuclear and cytoplasmic, or only very few cells stained by p504s, 3) positive for PCa: cytoplasmic staining of several epithelial cells (at least 20 cells with clear cytoplasmic staining) and no nuclear staining, and 4) non contributory (lack of cells, staining unclear, patchy).

Sometimes more than one slide were stained, depending on the amount of material. Furthermore, a standard report was elaborated with the following items:

Slide Nr \#: p63 \#, p504s \#,

Conclusion: \# of arguments in favour/against PCa; Suspicious for PCa, not contributory

This report is easy to fill out and can be done very quickly, this allows the report to be released in minutes after the interpretation in the electronic patient's document. 


\section{Results}

129 patients were enrolled in this study. Their age was from $47-81$ years, mean 66.2 years (median 67 years). Median PSA at biopsy was $7 \mathrm{ng} / \mu \mathrm{L}$ (range: 1.24-241).

Fifty one $(39.5 \%)$ patients had a prior history of $\mathrm{PCa}$, they were followed via active surveillance or focal therapy. The aim was to see whether their PCa had increased its aggressiveness or whether they displayed progression (bigger tumor size, more positive sites). Their initial tumor was either undetectable on MRI or $<5 \mathrm{~mm}$.

Examples of results of immunocytology are present in Figure 1 and 2. The immunocytological staining worked well, and with the staining of the cytoplasm by p504s, the cells were most of the time easy to diagnose as PCa cells (Fig.1). Frequently several basal cells weren't stained by p63, the basal cell marker. However, basal cells were easy to recognize by their round regular nuclear shape, and even if only some basal cells were stained, this excluded most of the time the diagnosis of PCa by lack of p504s supplementary staining (Fig. 2). And indeed, among the 129 patients, only one (0.8\%) had a non contributory cytology and was excluded from the statistical analyses.

On cytological examination (Table 1, Figure 3), 73 (57.0\%) of the 128 included patients were considered having PCa. Amongst them 68 (53.1\%) results were concordant with positive histology findings (PCa). Five cases were false positive (3.1\%). In $28(21.9 \%)$ cases, cytology results and histology results were negative for PCa, 17 (13.3\%) cases were considered negative in cytology and positive in histology, considered as false negative. Amongst them, $8(6.3 \%)$ cases concerned Gleason score $6(3+3)$ the best differentiated PCa. A total of $75 \%$ of results were identical in cytology and histology.

In our series, 10 (7.8\%) cytology samples were found suspicious for PCa, amongst them 8 $(6.3 \%)$ were positive for PCa, the biopsy specimen showed ISUP grade group 1-3. Two $(1.6 \%)$ cases were negative. If these suspicious cytologies were considered as positive for $\mathrm{PCa}$, a total of $81.3 \%$ of results were concordant between cytology and histology $(81.7 \%$ of the positive samples and $80.0 \%$ of the negative ones). The sensibility, specificity, predictive positive value and predictive negative value for cytology were $81.7 \%, 80.0 \%, 91.6 \%$ and $62.2 \%$, respectively. The strength of the association between cytology and histology was very high, with a Yule's coefficient of 0.89 . The $p$ value between cytology and histology was $<0.001$, showing no statistical difference between them. However, we were unable to systematically compare the biopsy sample on which cytology was done and the cytology sample. 
From a cytological point of view, it was impossible in case of a positive result to add values on the differentiation.

On the histological samples, 93 patients finally displayed $\mathrm{PCa}$. Among these patients, 40 $(40.3 \%)$ were considered to be in the intermediate group, with either Gleason score $7(3+4)$ or Gleason score $7(4+3)$. Twenty-nine patients $(31.2 \%)$ belonged to the low risk group (ISUP grade group 1) and $22(23.7 \%)$ to the high risk group.

When comparing the results of cytology and histology differentiation according to aggressiveness based on ISUP grade groups, (Table 2, Figure 4), cytology was able to detect $\mathrm{PCa}$ in $72.4 \%, 90.0 \%$ and $81.8 \%$ for the low, intermediate and high group, respectively. The best performances were thus observed for the most aggressive groups. 


\section{Discussion}

The handling of $\mathrm{PCa}$ is a major concern in public health and the importance of reference centres must be underlined (2). It allows a smooth follow-up and perfect handling of patients, as a good coordination with all actors is guaranteed. Ideally an early decision making allows to be conservative and in case of progression to treat the cancer as soon as possible (5).

Our approach of detecting and follow-up of PCa is entirely new, and our team is the first one to explore prostate cytology and immunocytology. It must be underlined that there exists need of expertise in cytology interpretation, because it is unusual and difficult, reading the slides might take some time. The time for reading a immuncytology slide is longer than reading a prostate biopsy, but globally within 5 minutes the immunocytology can be interpreted and the results can be given immediately.

The psychological impact of a PCa diagnosis is important and partial cultural differences exist. For instance in the northern countries, active surveillance is already a well-known option, but in southern countries like France, people still preferred being treated by radical prostatectomy (6). A recent study compared the psychological impact of favourable-risk prostate cancer (PCa) and associated treatment (active surveillance or radical treatment), assessing prevalence and temporal variability of generalized anxiety, PCa-specific anxiety and depression symptoms. Men undergoing active surveillance had significantly higher anxiety symptoms than those with radical treatment contradicting previous literature. Maybe this is due to a perceived inactivity of active surveillance relative to traditional narratives of cancer treatment. Therefore, the authors suggest to improve diagnosis and treatment information provision particularly for lower risk patients (7).

To our knowledge, this is the first study to investigate immuncytology and PCa detection in one day. Our results showed a concordance of $80.8 \%$ given within three hours. False positive cases concern less than $4.0 \%$ and false negative $13.6 \%$. In our study, 10 suspicious results also existed. In this case, the pathologist was not a $100 \%$ sure about the presence of tumor cells. Two cases were considered as false suspicious/positive as they were histologically negative. All the other eight cases displayed cancer.

One of the limitation of the study was to get used to the interpretation of slides which asked for some experience. Therefore, the interpretation by a uropathologist was an advantage, especially for the interpretation of the immunostaining. On the other hand, no architectural changes like in histology could be observed, the aspect on the slide was most of the time like small nests, sometimes close to a smear or to a real cytology with single cells. 
Although histology is the gold standard in detecting $\mathrm{PCa}$, the cytological approach can be interesting, especially if it is evident (Fig. 5). In this case, the result can be given immediately allowing to start a plan how to treat the patient immediately. Although MRI is a good tool to detect PCa, in case of small lesions mpMRI can not detect PCa (8). Furthermore, mimickers of PCa exist on MRI such as huge foci of atrophy, adenomatosis or basal cell hyperplasia (9). These lesions can increase the PSA levels, therefore histological or cytological cancer confirmation is absolutely necessary in order to treat the right patient.

Another problem of diagnosis and especially in cytology is the Gleason score $6(3+3)$ ISUP grade group 1. This well differentiated PCa can be difficult to diagnose even in histology. In histological specimen, the tumor cells sometimes do not homogenously express p504s, which can make the diagnosis difficult. In homogeneous loss of p63 is sometimes also seen in high grade PIN or atrophy. Sometimes it can be impossible even on histological samples to make the difference between PCa and no PCa and the pathologist has to classify the lesion as a focus of atypical glands/lesion suspicious for adenocarcinoma/ atypical small acinar proliferation or suspicious for cancer (10). These findings can also be seen in immuncytology. The highest false negative rate of our study was in well differentiated PCa samples with Gleason score $6(3+3)$ with $28.6 \%$ of false negative cases.

One of a probable bias of our study was that we did not always compare the cytology result with the same biopsy of which the cytology was taken. This might be one of the reasons why our results show only $80.8 \%$ of concordance. It is absolutely possible that cytology was made on a negative prostate biopsy and therefore, the cytology result was also negative, but we can not exclude that the patient didn't have another biopsy displaying PCa. Further studies will allow to refine these results. This study was a first trial of feasibility, to see whether it was possible to give quick results via immuncytology, shunting the pathology part which needs more time.

Although some biases exist, the results were satisfying for a first feasibility study in our department. The next step will be to compare the cytology result not with the globality of prostate biopsies, but with the sample on which the biopsy has been made. A global concordance of $80.8 \%$ is a good start to continue our way, but further studies with higher sample numbers are requested.

No funding 


\section{References}

1. Heidenreich A, Bastian PJ, Bellmunt J, Bolla M, Joniau S, van der Kwast T, et al. EAU guidelines on prostate cancer. part 1: screening, diagnosis, and local treatment with curative intent-update 2013. Eur Urol.;65(1):124-37.

2. Wirth M, Fossati N, Albers P, Bangma C, Brausi M, Comperat E, et al. The European Prostate Cancer Centres of Excellence: A Novel Proposal from the European Association of Urology Prostate Cancer Centre Consensus Meeting. Eur Urol. 2019;76(2):179-86.

3. Berman A, Teig B, Duracinsky M, Gayet M, Bellin M-F, Guettier C, et al. [One day diagnosis for breast lesions: Medical and psychological assessment--EVADIASEIN study]. J Gynecol Obstet Biol Reprod (Paris). 2016;45(1):21-8.

4. Epstein JI, Egevad L, Amin MB, Delahunt B, Srigley JR, Humphrey PA, et al. The 2014 International Society of Urological Pathology (ISUP) Consensus Conference on Gleason Grading of Prostatic Carcinoma: Definition of Grading Patterns and Proposal for a New Grading System. Am J Surg Pathol. 2016;40(2):244-52.

5. Mottet N, Bellmunt J, Bolla M, Briers E, Cumberbatch MG, De Santis M, et al. EAUESTRO-SIOG Guidelines on Prostate Cancer. Part 1: Screening, Diagnosis, and Local Treatment with Curative Intent. Eur Urol. 2017;71(4):618-29.

6. Adam S, Feller A, Rohrmann S, Arndt V. Health-related quality of life among long-term ( $\geq 5$ years) prostate cancer survivors by primary intervention: a systematic review. Health Qual Life Outcomes. 2018;16(1):22.

7. Ruane-McAteer E, Porter S, O’Sullivan J, Dempster M, Prue G. Investigating the psychological impact of active surveillance or active treatment in newly diagnosed favorable-risk prostate cancer patients: A 9-month longitudinal study. Psychooncology. 2019;28(8):1743-52.

8. Johnson DC, Raman SS, Mirak SA, Kwan L, Bajgiran AM, Hsu W, et al. Detection of Individual Prostate Cancer Foci via Multiparametric Magnetic Resonance Imaging. Eur Urol. 2019;75(5):712-20.

9. Kitzing YX, Prando A, Varol C, Karczmar GS, Maclean F, Oto A. Benign Conditions That Mimic Prostate Carcinoma: MR Imaging Features with Histopathologic Correlation. Radiogr Rev Publ Radiol Soc N Am Inc. 2016;36(1):162-75.

10. Van der Kwast T, Bubendorf L, Mazerolles C, Raspollini MR, Van Leenders GJ, Pihl C$\mathrm{G}$, et al. Guidelines on processing and reporting of prostate biopsies: the 2013 update of the pathology committee of the European Randomized Study of Screening for Prostate Cancer (ERSPC). Virchows Arch Int J Pathol. 2013;463(3):367-77. 


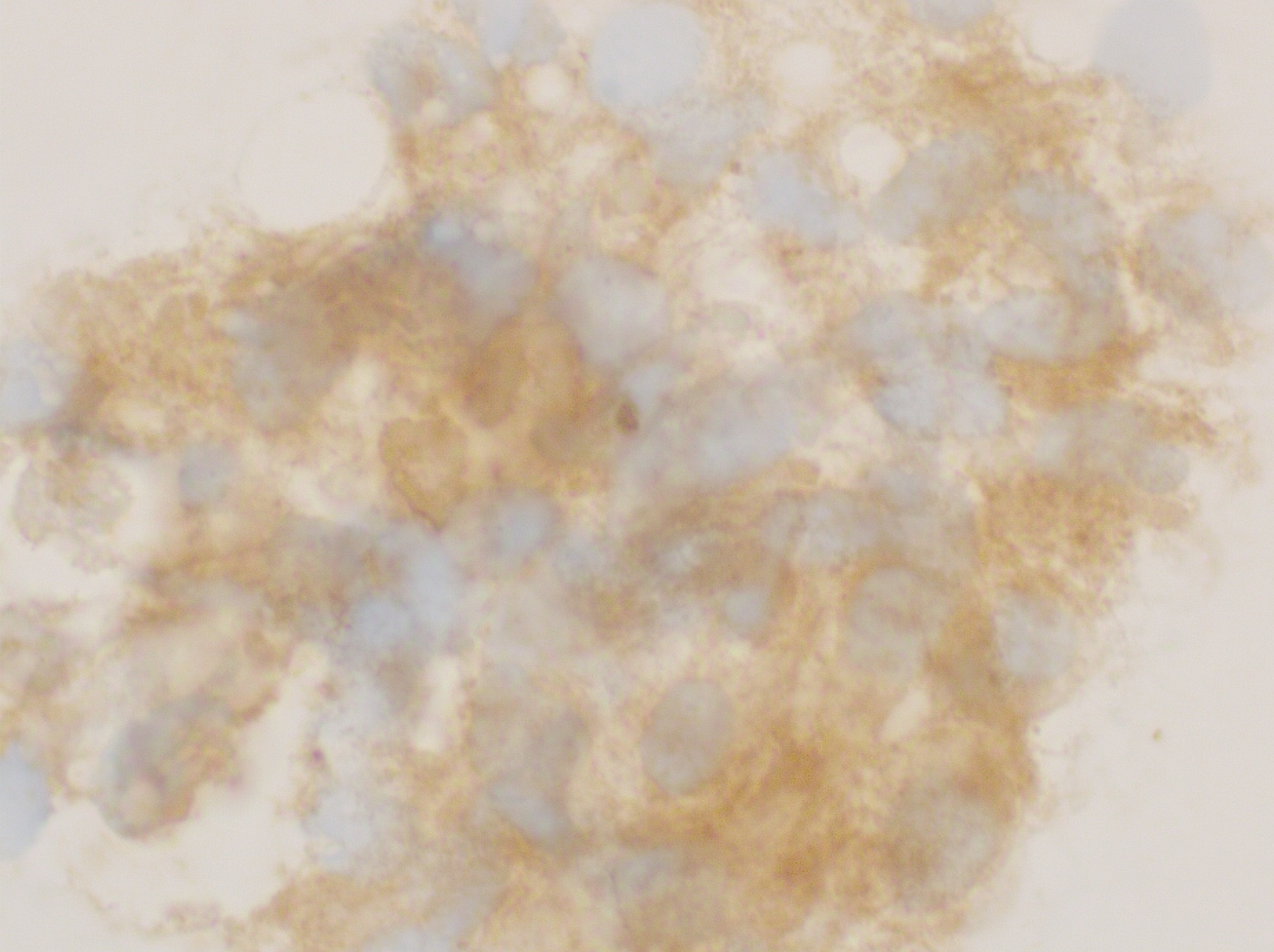




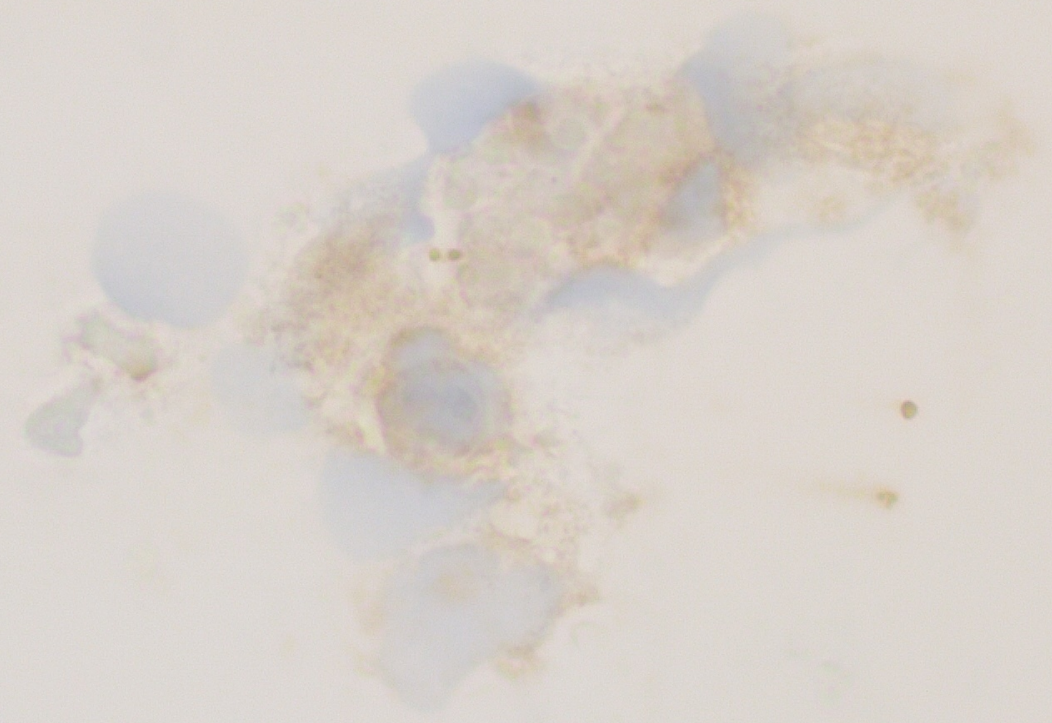




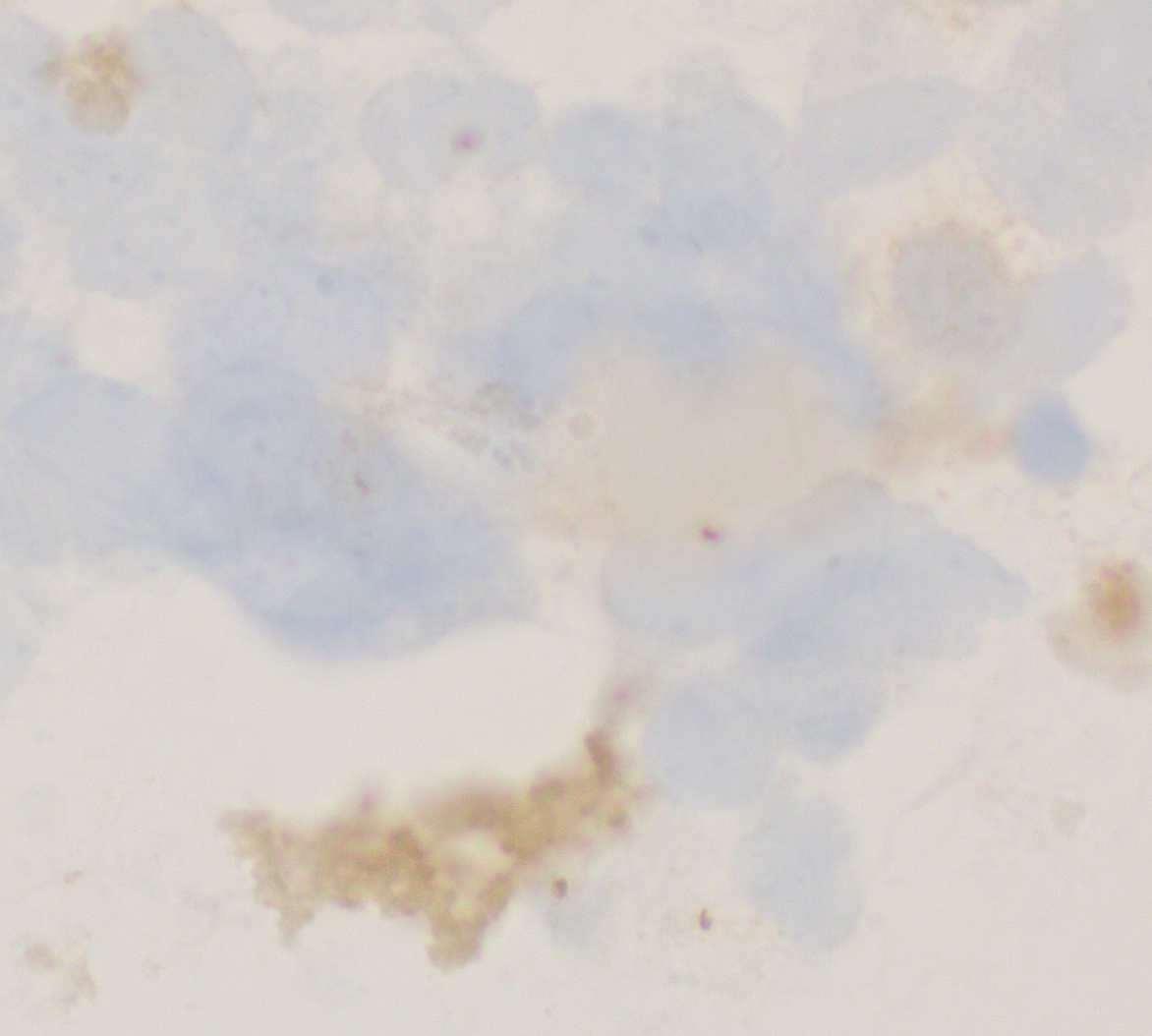



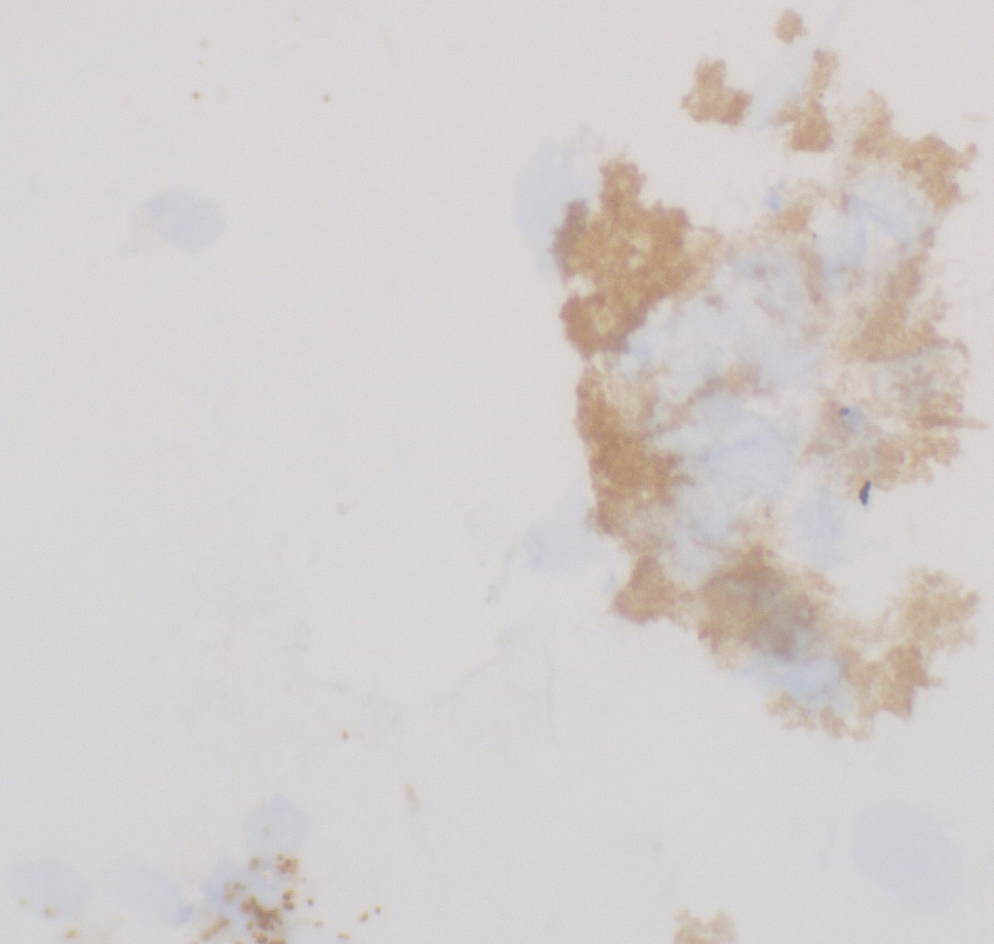

wit 